\title{
Hydrodynamic correlations in the translocation of a biopolymer through a nanopore: Theory and multiscale simulations
}

\section{Citation}

Fyta, Maria, Simone Melchionna, Sauro Succi, and Efthimios Kaxiras. 2008. "Hydrodynamic Correlations in the Translocation of a Biopolymer through a Nanopore: Theory and Multiscale Simulations." Physical Review E 78 (3). https://doi.org/10.1103/physreve.78.036704.

\section{Permanent link}

http://nrs.harvard.edu/urn-3:HUL.InstRepos:41384104

\section{Terms of Use}

This article was downloaded from Harvard University's DASH repository, and is made available under the terms and conditions applicable to Open Access Policy Articles, as set forth at http:// nrs.harvard.edu/urn-3:HUL.InstRepos:dash.current.terms-of-use\#OAP

\section{Share Your Story}

The Harvard community has made this article openly available. Please share how this access benefits you. Submit a story. 


\title{
Hydrodynamic correlations in the translocation of biopolymer through a nanopore: theory and multiscale simulations
}

\author{
Maria Fyta ${ }^{1}$, Simone Melchionna ${ }^{2,3}$, Sauro Succi ${ }^{4,5}$ and Efthimios Kaxiras ${ }^{1,2}$ \\ ${ }^{1}$ Department of Physics and ${ }^{2}$ School of Engineering and Applied Sciences, Harvard University, Cambridge, MA, USA \\ ${ }^{3}$ INFM-SOFT, Department of Physics, Università di Roma La Sapienza, P.le A. Moro 2, 00185 Rome, Italy \\ ${ }^{4}$ Istituto Applicazioni Calcolo, CNR, Viale del Policlinico 137, 00161, Roma, Italy \\ ${ }^{5}$ Initiative in Innovative Computing, Harvard University, Cambridge, MA, US
}

(Dated: October 26, 2018)

\begin{abstract}
We investigate the process of biopolymer translocation through a narrow pore using a multiscale approach which explicitly accounts for the hydrodynamic interactions of the molecule with the surrounding solvent. The simulations confirm that the coupling of the correlated molecular motion to hydrodynamics results in significant acceleration of the translocation process. Based on these results, we construct a phenomenological model which incorporates the statistical and dynamical features of the translocation process and predicts a power law dependence of the translocation time on the polymer length with an exponent $\alpha \approx 1.2$. The actual value of the exponent from the simulations is $\alpha=1.28 \pm 0.01$, which is in excellent agreement with experimental measurements of DNA translocation through a nanopore, and is not sensitive to the choice of parameters in the simulation. The mechanism behind the emergence of such a robust exponent is related to the interplay between the longitudinal and transversal dynamics of both translocated and untranslocated segments. The connection to the macroscopic picture involves separating the contributions from the blob shrinking and shifting processes, which are both essential to the translocation dynamics.
\end{abstract}

\section{INTRODUCTION}

Translocation of biopolymers, such as DNA and RNA, plays a vital role in many important biological processes, such as viral infection by phages, inter-bacterial DNA transduction or gene therapy [1]. This has motivated a number of in vitro experimental studies, aimed at exploring the translocation process through protein channels across cellular membranes [2, 3], or through microfabricated channels [4]. In particular, recent experimental work has focussed on the possibility of fast DNAsequencing by "reading-off" the DNA bases while tracking its motion through nanopores under the effect of a localized electric field [ $\underline{5}, \underline{6}$ ].

The translocation of biopolymers is a complex phenomenon involving competition between many-body atom-atom interactions, fluid-atom hydrodynamic coupling, as well as the interaction of the polymer with wall molecules in the nanopore. Although some universal features of the translocation process can be analyzed by means of suitably simplified statistical models [7, 8, 9, 10], and non-hydrodynamic coarse-grained or microscopic models [11, 12, 13, 14], a quantitative description of this complex phenomenon calls for realistic, stateof-the-art computational modeling. Work along these lines has been recently reported by several groups, beginning with the first multiscale simulations by the present authors 15, 16], followed by Langevin dynamics simulations [17] and more recently by coupled molecular-fluid dynamics [18, 20]. Specifically, Forrey and Muthukumar performed Langevin dynamics simulations, and examined single-file as well as multi-file translocation [17]. Izmitli et al. used a coupled lattice Boltzmann - Molecular Dynamics scheme [18], as outlined in [15, 16], and reproduced our early results, while exploring a smaller range of chain lengths and ensemble size. These recent works have provided a wealth of new computational results and detailed insight into the problem of translocation through nanopores. In addition to these studies, Slater and co-workers have investigated the translocation process numerically by also including hydrodynamic interactions [19]. However, these authors treat the cases were no external field is applied, thus comparison with our work cannot be made at this point.

In this work, we report a synthesis of the simulational result into a coherent mean-field analytical model which captures the basic physical mechanisms behind the translocation process. The model is based on extracting the scaling behavior of translocated and untranslocated segments, including the anisotropy between longitudinal and transverse components. The analytical model predicts a power-law scaling behavior of translocation time with polymer length with exponent $\alpha \approx 1.2$, which is very close to the one found in the current $(\alpha=1.28 \pm 0.01)$ and other $(\alpha=1.28 \pm 0.03[18])$ simulations and in experiments of DNA translocation $(\alpha=1.27 \pm 0.03[\underline{6}])$.

The paper is organized as follows: in Section II we provide an overview of our simulational approach. In Section III we discuss the choice of simulation parameters that make our simulations relevant to DNA translocation through nanopores and the implications for the implied time-scales and length-scales of the system. In Section IV we discuss the results of the translocation simulations, paying particular attention to the anisotropy of longitudinal and transverse components of the translocating polymer in both the untranslocated and translocated segments. Section V presents the analytical meanfield model and its comparison to the simulations. We conclude in Section VI with some comments on what may be the limitations of the mean-field picture and a 
summary of our results.

\section{MULTISCALE MODEL}

Our multiscale method is based on the coupling between constrained Molecular Dynamics (MD) for the polymer evolution and a lattice Boltzmann (LB) treatment of the explicit solvent dynamics [21, 22]. In contrast to Brownian dynamics, the LB approach handles the fluid-mediated solute-solute interactions through an explicit representation of local collisions between the solvent and solute molecules. We will focus on the fast translocation regime, in which the translocation time $\tau$ is much smaller than the typical relaxation (Zimm) time of the polymer towards its native (minimum energy, maximum entropy) configuration. This regime cannot be captured by a simple, one-dimensional Brownian model [23] or a Fokker-Planck representation.

Translocation is induced by the constant electric field $\vec{E}$ acting along the $x$ direction localized in a region near the pore. The dynamics of the beads which constitute the molecule are governed by the equation

$$
m_{b} \frac{d \vec{v}_{i}}{d t}=\vec{F}_{t o t, i}=\vec{F}_{c, i}+\vec{F}_{d r a g, i}+\vec{F}_{r, i}+\vec{F}_{\kappa, i}+\vec{F}_{d r i v e, i}
$$

with $\vec{F}_{t o t, i}$ the total force on bead $i . \vec{F}_{c, i}$ is a conservative force describing the sum of bead-bead and bead-wall interactions; $\vec{F}_{d r a g, i}$ is the dissipative drag force due to polymer-fluid coupling given by $-m_{b} \gamma\left(\vec{v}_{i}-\vec{u}_{i}\right)$ with $\gamma$ the friction coefficient and $\vec{v}_{i}, \vec{u}_{i}$ the bead and fluid velocities at the position $\vec{r}_{i}$ of bead $i$ with a mass $m_{b} ; \vec{F}_{r, i}$ is a random force on bead $i$ with zero mean; $\vec{F}_{\kappa, i}$ is the reaction force resulting from $N_{0}-1$ holonomic constraints for molecules modelled with rigid covalent bonds, with $N_{0}$ the number of beads in the polymer; and $\vec{F}_{\text {drive }, i}$ is the driving force representing the effect of the external field $\vec{E}$, equal to $q \vec{E} g\left(\vec{r}_{i}\right)$ with $q$ an effective charge, which acts only on beads in the pore region. The region over which the external field acts is described by the function $g\left(\vec{r}_{i}\right)$, which is 1 for $\vec{r}_{i}$ within this region and 0 otherwise; the extent of this region is chosen to be a cube of side $3 \Delta x$ (shown by the green shaded region in Fig. 11), where $\Delta x$ is the lattice spacing.

For the bead-bead interaction, other than that between consecutive beads, and for the bead-wall interaction we choose separately a truncated Lennard-Jones potential (repulsive part only [28]):

$$
V(r)= \begin{cases}4 \varepsilon\left[\left(\frac{\sigma}{r}\right)^{12}-\left(\frac{\sigma}{r}\right)^{6}\right] & \text { if } r \leq r_{c u t} \\ 0 & \text { if } r>r_{c u t}\end{cases}
$$

In this expression, $r$ is the bead-bead or bead-wall distance. In both cases the potentials are truncated at the cut-off distance $r_{c u t}=2^{1 / 6} \sigma$. The chosen parameters $\sigma$ and $\varepsilon$ are $1.8 \Delta x$ and $10^{-4} \Delta m \Delta x / \Delta t^{2}$ for the beadbead interactions and $1.5 \Delta x$ and $10^{-4} \Delta m \Delta x / \Delta t^{2}$ for

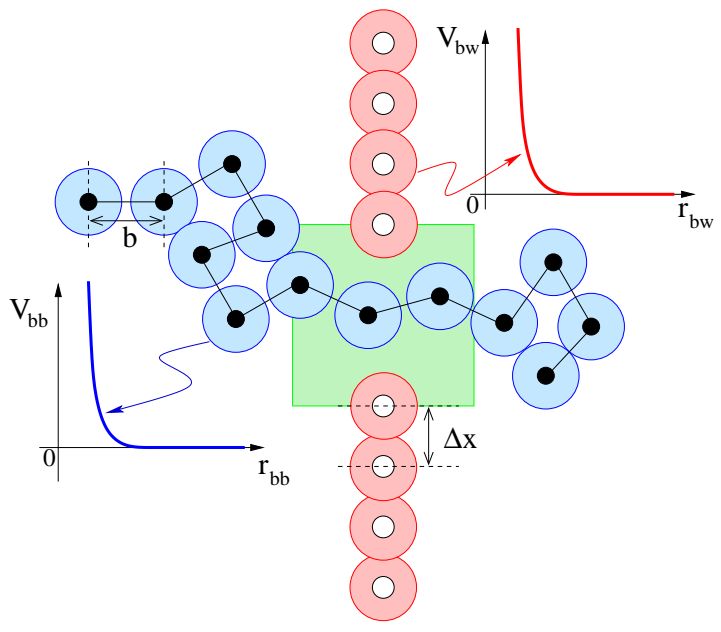

FIG. 1: (Color online) Illustration of the interactions in the DNA translocation model: the beads representing the DNA are shown as small black dots connected by straight line segments, the wall is represented by a plane of on-lattice points (small white dots separated by the lattice spacing $\Delta x$ ) which repel the DNA beads within a range of interaction. The beadbead interactions are indicated by the blue spheres surrounding the small black ones, defined in Eq.(2), except for the distance between consecutive beads which is fixed at $b$. Interactions between the beads and the wall are indicated by the red spheres surrounding the small white ones, defined in Eq. (2). Interaction between the beads and the constant external field are confined over a shaded (in green) region around the pore (see text for details).

the bead-wall interactions. The parameters are given in LB units as explained in Section III. The distance between consecutive beads along the chain representing the macromolecule is set to $b=1.2 \Delta x$ through a constraint imposed by the SHAKE algorithm [24]. The geometry of the pore region is shown schematically in Fig. 1] The fluid is represented through lattice Boltzmann particles that reside on a three-dimensional cubic lattice with spacing $\Delta x$. The probability distribution $f_{p}(\vec{x}, t)$ denotes the number of particles at the lattice position given by $\vec{x}$ at time $t$, and evolves in space and time toward the equilibrium distribution $f_{p}^{e q}$ and with relaxation frequency $\omega$, as:

$$
\begin{aligned}
f_{p}\left(\vec{x}+\vec{c}_{p} \Delta t, t+\Delta t\right)= & f_{p}(\vec{x}, t)-\omega \Delta t\left(f_{p}-f_{p}^{e q}\right)(\vec{x}, t) \\
& +F_{p} \Delta t+G_{p} \Delta t
\end{aligned}
$$

The LB particles can only move on the lattice with fixed first- and second-neighbor speeds $\vec{c}_{p}$ (19 in all, for a 3D cubic lattice), while $F_{p}$ represents thermal fluctuations and $G_{p}$ describes the polymer-fluid back reaction

$$
G_{p}(\vec{x}, t)=w_{p} \beta \sum_{i \in D(x)}\left[\vec{F}_{d r a g, i}+\vec{F}_{r, i}\right] \cdot \vec{c}_{p}
$$

with $w_{p}$ a set of weights normalized to unity, and $\beta$ the inverse fluid temperature. Finally, $D(x)$ denotes the lattice cell to which the $i^{t h}$ bead belongs. Details on the 
numerical implementation of the scheme have been reported in Ref. 15.

Compared to other numerical methods, the present lattice Boltzmann-Molecular Dynamics (LB-MD) scheme has certain computational advantages, namely, it permits to take into account self-consistent hydrodynamic correlations at computational cost scaling linearly with the polymer length. This allowed us to simulate long chains in 3D over large statistical ensembles at an affordable computational cost. We simulate polymers of various sizes and as large sample realizations as our computational resources permit; specifically we considered sizes of $N_{0}=20$ [1000], $N_{0}=50$ [1000], $N_{0}=100$ [500], $N_{0}=200$ [300], $N_{0}=300$ [300], $N_{0}=400$ [200], $N_{0}=500$ [150], where the numbers in brackets are the sample realizations.

\section{CHOICE OF THE SIMULATION PARAMETERS}

We discuss next the details of the choice of parameters in the model so that our simulations are relevant to DNA translocation through nano-pores, as observed in typical experimental setups [6]. The simulations are performed in a three-dimensional box which contains the polymer and the fluid solvent, and has a size $N_{x} \times N_{x} / 2 \times N_{x} / 2$ in units of the lattice spacing $\Delta x$; we used $N_{x}=80$ for polymers of size $N_{0} \leq 400$ beads. The separating wall is located in the mid-section of the $x$ direction, at $x / \Delta x=N_{x} / 2$, with a square hole of side $3 \Delta x$ at the center through which the polymer translocates from one chamber to the other. At $t=0$ the polymer resides entirely in the right chamber at $x / \Delta x>N_{x} / 2$, with its one end at the pore region along the streamline $(x)$ and cross-flow $(y, z)$ directions. The LB time step is $\Delta t$ and the MD time step $\Delta t / 5$.

In order to have a plausible representation of DNA, we choose the separation between consecutive beads to be $b=50 \mathrm{~nm}$, the persistence length of double-stranded DNA. Since we defined $b=1.2 \Delta x$, this gives for the lattice spacing $\Delta x=42 \mathrm{~nm}$. Since the repulsive interaction between the beads and the wall involved the parameter $\sigma_{w}=1.5 \Delta x$, and the pore is a square of size $3 \Delta x$, this produces an effective hole of size $\sim 5 \mathrm{~nm}$ through which the polymer translocates. Having set the value of $\Delta x$, we choose the time step so that the kinematic viscosity is expressed as:

$$
\nu_{w}=\nu_{L B} \frac{(\Delta x)^{2}}{\Delta t},
$$

with $\nu_{w}$ the viscosity of water $\left(10^{-6} \mathrm{~m}^{2} / \mathrm{s}\right)$ and $\nu_{L B}$ the numerical value of the viscosity in LB units; taking $\nu_{L B}=$ 0.1 produces a time-step of $\Delta t=160 \mathrm{ps}$.

The above choice for the lattice spacing and timestep fixes the lattice speed $c \equiv(\Delta x / \Delta t) \sim 250 \mathrm{~m} / \mathrm{s}$, which is reasonably close to the solvent thermal speed $\sqrt{k T / m_{w}} \sim 500 \mathrm{~m} / \mathrm{s}$. It is instructive to compare the lattice speed, $c$, with the typical propagation speeds of the main phenomena of interest in the translocation process. These are:

i) the translocation speed $b(d N / d t) \sim 10^{-3} \mathrm{~m} / \mathrm{s}$;

ii) the electric drift speed $(q E) /\left(m_{b p} \gamma_{b p}\right) \sim 0.3 \mathrm{~m} / \mathrm{s}$;

iii) the base-pair thermal speed $\sqrt{k T / m_{b p}} \sim 70 \mathrm{~m} / \mathrm{s}$, where we have used the following reference values for the base-pair translocation rate $d N / d t \sim 10^{7} \mathrm{bp} / \mathrm{s}$, the strength of the force due to the external field $q E \sim 10$ $\mathrm{pN}$, the mass of a base-pair $m_{b p}$ in terms of the mass of the water molecule $m_{w}, m_{b p} \sim 30, m_{w} \sim 600 \mathrm{amu}$, the drag coefficient for a base-pair $\gamma_{b p}=3 \times 10^{13} \mathrm{~s}^{-1}$ [29] and $T=300{ }^{0} \mathrm{~K}$ for the temperature. These orderof-magnitude estimates indicate that the present choice of space and time units is such that the corresponding speed, $u$, fulfills the numerical stability CFL (CourantFriedrichs-Lewy) condition, $u<c$.

In LB simulations mass units are fixed by the mass density of the fluid species $\rho_{w}$. Setting the LB mass density $\rho_{L B}=1.0$ corresponds to having a number of water molecules $\rho_{w} \Delta x^{3} / \rho_{L B} m_{w}=2 \times 10^{6}$, where $\rho_{w}$ is the density of water, and each lattice site contains a solvent mass $\Delta m=\rho_{w} \Delta x^{3} / \rho_{L B}$. Since we are using a continuum-kinetic representation of fluid flow at the nanoscale, a necessary condition for this representation to hold against statistical noise is that the above ratio be much greater than unity. Given the fact that we have 19 discrete distributions, $f_{i}$ per cell, each of them would represent about $10^{5}$ water molecules, a safe value towards satisfying this condition.

The prime goal of the mapping procedure is to secure the correct values of the major dimensionless parameters governing the physics of the translocation process. In particular, this regards the ratio of external drive to thermal forces which we will call $\phi$, defined as

$$
\phi \equiv \frac{q E b}{k T}
$$

The value of this quantity in actual experiments is $\phi \sim$ $1-10$ [6]. In our simulations, we took $k T=10^{-4}$ and $q E=0.01$ acting on one bead, mapping $\simeq 100$ basepairs. These quantities are again given in LB units. At a base-pair level, this means $q E=10^{-4}$, corresponding to $\phi=1$, in satisfactory order-of-magnitude agreement with experiments. With this value of the driving force and the polymers studied in the present work, a typical translocation event takes place in a time interval on the order of $10^{3}-10^{4} \mathrm{LB}$ timesteps.

In the simulations we define the effective mass of the beads to be $m_{b}=1$. A straightforward calculation shows that the ratio of the effective bead mass to the mass resulting from the DNA coarse graining, that is, one bead representing $\sim 100$ base pairs, is $\sim 700$. On the other hand, the parameter relevant to momentum exchange is the bead friction $\gamma$. We chose $\gamma=0.1$ in LB units, so that the ratio between friction in physical units and the experimentally determined one $\left(\gamma_{b p}=30 \mathrm{ps}^{-1}\right.$ [29] $)$ is $10^{-4}$. Our choice was dictated by the criterion of numerical stability $\gamma<1 / \Delta t=6 \times 10^{9} \mathrm{~s}^{-1}$. These fac- 


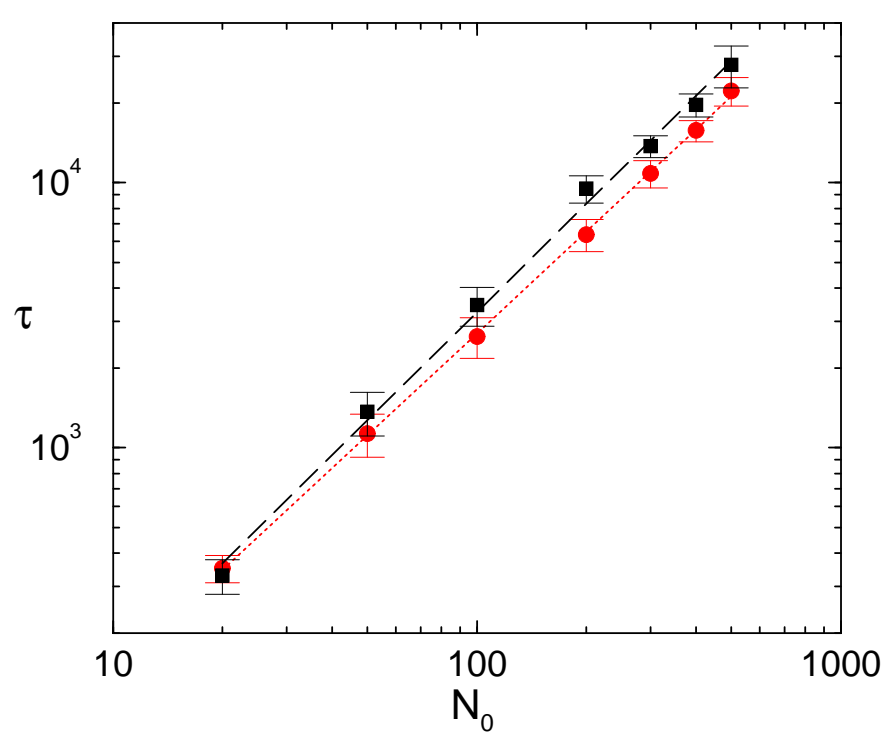

FIG. 2: (Color online) Scaling of the translocation time $\tau$ with the number of beads $N_{0}$, in the presence (circles) or absence (squares) of coupling between the molecule and the solvent. The exponents are $1.28 \pm 0.01$ and $1.36 \pm 0.03$, respectively.

tors taken together show that the bare particle mobility, $\mu=1 /\left(m_{b} \gamma\right)$, is a factor of 15 larger than the experimental one. This is equivalent to an underdamped motion of the macromolecule, which results in smooth particle trajectories and allows for algorithmic stability without affecting the long-time behavior of the polymer. A possible alternative would be to solve the polymer dynamics in overdamped (Brownian) form which also circumvents the small timestep issue imposed by the frictional damping [26, 27]. Our choice to use inertial dynamics was based on previous experience with similar systems, without hydrodynamic interactions, which showed that the inertial dynamics approach has a slight advantage in stability with larger time-step size.

\section{TRANSLOCATION SIMULATIONS}

We report next the results of extensive simulations of translocation events. In each case, the translocation time exhibits a statistical (not exactly gaussian) distribution around the most probable translocation time, in close analogy to experimental observations $[2,3,6]$. According to these studies, a $48.5 \mathrm{kbp}$ double-stranded DNA takes about $2 \mathrm{msec}$ to translocate, while a typical Zimm time is about 700 msec. Fig. 2 shows the dependence of the most probable translocation time $\tau$ on the polymer length, which obeys a superlinear scaling relation: $\tau \propto N_{0}^{\alpha}$. The observed exponent, with the moleculefluid coupling in operation, $\alpha \simeq 1.28 \pm 0.01$ is in very good agreement with recent experiments of DNA translocation through a nanopore driven by an external electric field, where $\alpha \simeq 1.27 \pm 0.03[\underline{6}]$.

In the absence of coupling between the molecule and the solvent, the translocation process is slowed down, as indicated by a higher exponent, $\alpha \simeq 1.36 \pm 0.03$. We propose that in the presence of polymer-solvent coupling some form of bead-bead screening takes place, the biopolymer never translocates in the form of a linear chain, as this configuration is entropically suppressed. The physics of the larger exponent (slower motion) in the absence of hydrodynamics is related to enhanced correlation effects and a wider range of polymer fluctuations.

In Fig. [3 we show an ensemble of 100 polymer configurations at three different instants, referring to the initial, mid-point and final stages of translocation. From this figure it is clear that, initially, the shape of the untranslocated (U) segment is squeezed against the wall and takes the form of an oblate ellipsoid. The translocated (T) segment appears to be more compact and prolate. The tendency of the U-segment to be attracted to the wall is in line with the well-known "mushroom" shape arising from a polymer anchored at one end to a repulsive wall 31, 32]. To investigate the consequences of this anisotropy, we have inspected the scaling of the gyration tensor with the number of monomers for the $\mathrm{U}$ and $\mathrm{T}$ segments separately, with time, according to:

$$
R_{I, \mu}(t) \propto\left[N_{I}(t)\right]^{\nu_{I, \mu}},(I=U, T ; \mu=\perp, \|)
$$

where $\mu=\|, \perp$ denotes the longitudinal and transverse components of the gyration tensor, with respect to the direction of translocation. This scaling is shown in Fig. 4. from which we observe that for $N_{U}>100$ the transverse component $R_{U, \perp}$ follows a dynamic scaling law with $\nu_{U, \perp} \simeq 0.6$, close to the Flory-exponent of a 3 -D selfavoiding random walk, while the longitudinal one exhibits a much weaker dependence on $N_{U}$ (smaller slope). The T-segment follows a similar trend, but with a transverse component scaling with $\nu_{T, \perp} \simeq 0.5$.

The fluid/biopolymer system can be approached as an extended dynamical system consisting of two components: this system receives energy from the exterior through a localized electric field acting on the polymer and dissipates it via interaction of both the fluid and the polymer with the wall. Each bead is subject to the following forces: (a) the localized drive $\vec{F}_{\text {drive, } i}$, (b) the dissipative drag $\vec{F}_{\text {drag }, i}$, (c) the pore drag $\vec{F}_{\text {pore }, i}$, and (d) the entropic forces. At equilibrium, the latter can be expressed as

$$
F_{\text {entr }} \sim \frac{k_{B} T}{b} \frac{1-2 r}{r(1-r)}
$$

where $r(t) \equiv N_{T}(t) / N_{0}$ is the translocation coordinate [7] and $b$ is the separation between two beads. Here, we have used an explicit dependence on $r$ simply to show that entropic forces are negligible most of the time, except at both ends (initiation and completion) of the translocation process. These forces are naturally measured in terms of the thermal force, $F_{t h}=k_{B} T / b$. In the fast translocation 
regime considered in this work, $F_{d r i v e} / F_{t h}>1$, yielding $b F_{\text {drive }} / k_{B} T \sim 10^{2}$ for the parameters used. Pore forces are negligible in our simulations, due to the small pore size. For the entropic forces, $F_{\text {entr }} / F_{t h} \sim(1-2 r) /(r(1-$ $r)$ ), which shows that they can be neglected except at the early $(r(t) \rightarrow 0)$ and final $(r(t) \rightarrow 1)$ stages of the process. With entropic forces and pore dissipation negligible, the forces guiding the translocation are the hydrodynamic drag and the drive from the external electric field.

Hydrodynamics is expected to provide a cooperative background, helping to minimize frictional effects. For quantitative insight into this, we monitor the synergy factor, defined as the work per unit time made by the fluid on both parts $(I=U$ or $T)$ of the polymer:

$$
S_{H}^{(I)}(t)=\frac{d W_{H}^{(I)}}{d t}=\gamma\left\langle\sum_{i}^{N_{I}} \vec{v}_{i}^{(I)}(t) \cdot \vec{u}_{i}(t)\right\rangle
$$

where brackets denote averages over different realizations of the polymer for a given length. Positive (negative) values of $S_{H}^{(I)}(t)$ indicate a cooperative (competitive) solvent, respectively. The variation of $S_{H}^{(I)}(t)$ with time is linear (Fig. 5(a)), while the total rate $S_{H}(t)=$ $S_{H}^{(T)}(t)+S_{H}^{(U)}(t)$ on the whole chain, is constant with time indicating that the work per unit time associated with the change of the radii of the two blobs is constant. The probability distribution of $S_{H}(t)$ during translocation (Fig. [5(b)), indicates that hydrodynamics turns the solvent into a cooperative environment: the distribution lies entirely in the positive range. This cooperative effect is the underlying reason for the faster translocation process in the presence of hydrodynamic interactions. Similarly, the work done per timestep by the electric field on the polymer can be defined as:

$$
S_{E}(t)=\frac{d W_{E}}{d t}=\left\langle\sum_{i} \vec{F}_{d r i v e, i} \cdot \vec{v}_{i}(t)\right\rangle
$$

The average of $S_{E}(t)$ is positive (Fig. $5($ a)). However, the negative tail of the corresponding probability distribution in Fig. 国(b) indicates that there is a non-negligible probability to find beads moving against the electric field. On average, $S_{E}(t)$ is also constant with time (except very near the completion of the translocation) denoting that the beads traverse the pore with basically the same speed at all times. The average hydrodynamic work per time is larger than the input of the external electric field, since the latter only acts on a very small fraction of the beads, about 4 resident beads within the pore region.

\section{PHENOMENOLOGICAL MODEL}

We next present a phenomenological model for the scaling exponent of the translocation time in the presence of the solvent. We first consider Eq.(11) and multiply both sides of the equation with the bead velocity $\vec{v}_{i}$.

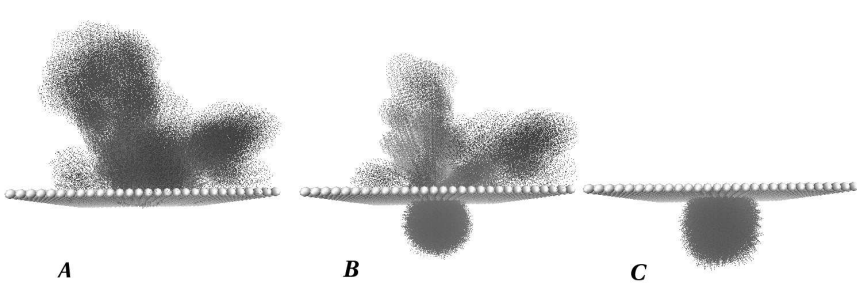

FIG. 3: 3-D view of an ensemble of 100 polymers with $N_{0}=$ 300 at different stages of the process: $A, B$ and $C$ correspond to the initial, mid-point, and final translocation times.

Summing up over all beads, using the fact that the velocities are uncorrelated with the random forces $\vec{F}_{r, i}$ and that $\vec{F}_{\kappa, i}$ produces no work, we obtain:

$$
\frac{d K}{d t}=\left(\frac{d W}{d t}\right)^{(1)}+\left(\frac{d W}{d t}\right)^{(2)}+S_{E}(t)
$$

where $K$ is the kinetic energy of the polymer and the right-hand side of this equation contains the change in energy of the $\mathrm{U}$ and $\mathrm{T}$ sections of the polymer due to the mechanical work $W^{(1)}$, the viscous drag, $W^{(2)}=S_{H}-$ $2 \gamma K$, and the rate of work done by the electric field, $S_{E}$. By writing Eq. (10) we assume that the translocation time $\tau\left(N_{0}\right)$ for the entire polymer is determined by two separate contributions. The first stems from the change in free energy of the polymer due to the removal of beads from the $\mathrm{U}$ side and their addition to the $\mathrm{T}$ side. The second term arises from shifting the center of mass of each blob from the initial position towards (for the U part), or away from (for the T part), the pore entrance.

The simulations reveal that $S_{H}$ and $S_{E}$ are each independently constant in time to a very good approximation. This holds for both the average values over all samples (see Fig. [5(a)) and for any polymer realization. The average $K$ (not shown) is also approximately constant, as the temperature is constant, leading to $d K / d t=0$.

Regarding the mechanical work $W^{(1)}$, simulation data show that, except for a short-lived transient at the beginning and final part of the process, the rate of removal/addition of beads is linear in time. Similarly, for the viscous drag the shift of the center of mass takes place at constant velocity, as illustrated in Fig. 5 by the two individual components $S_{H}^{(U)}(t)$ and $S_{H}^{(T)}(t)$, both of which show linear behavior. Since the two contributions can be viewed as independent components of the work, we separately analyze their effects on the scaling dependence.

In order to estimate the work required to shrink/grow the two blobs, we now introduce a macroscopic picture according to which, for a spherical blob of radius $R_{I}$, surface $A_{I}$ and volume $V_{I}$ the work is $d W_{I}^{(1)}=$ $P_{I} d V_{I}+\sigma_{\gamma} d A_{I}$ with $\sigma_{\gamma}$ the average surface tension and $P_{I}=2 \sigma_{\gamma} / R_{I}$ the pressure acting on the blob (Laplace equation). Given the anisotropy of the $I=U$ and 


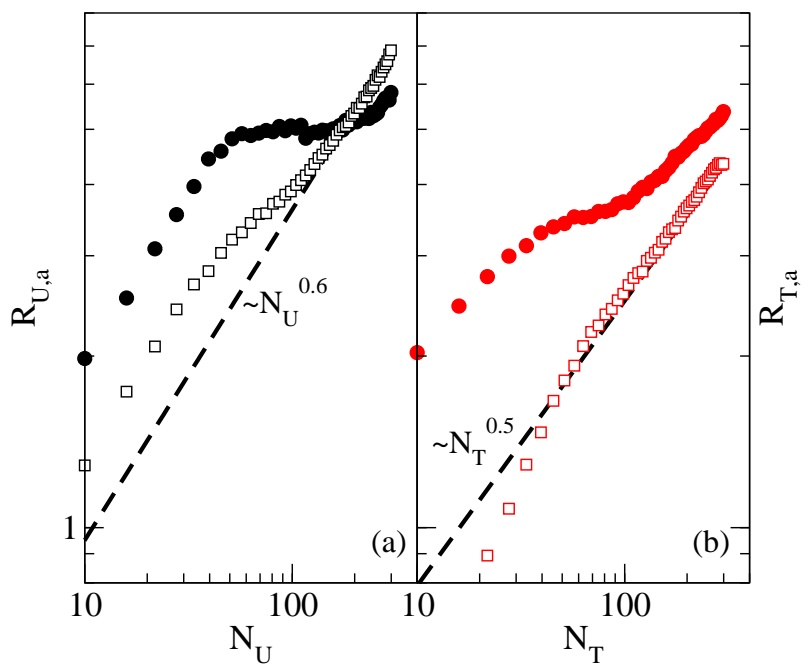

FIG. 4: (Color online) Longitudinal (filled circles) and transverse (open squares) components of the gyration tensor for (a) the $U$ and (b) the $T$ segment with the number of untranslocated $\left(\mathrm{N}_{U}\right)$ and translocated $\left(\mathrm{N}_{T}\right)$ beads, respectively. The dashed lines show the scaling.

$T$ segments, the above relation generalizes to $d W^{(1)}=$ $\sum_{I} \sum_{\mu=\perp, \|} \lambda_{I, \mu} R_{I, \mu} d R_{I, \mu}$ with $\lambda_{I, \mu}$ collecting all constants. More explicitly, $R_{U, \perp} d R_{U, \perp} \sim N_{U}^{2 \nu_{U}-1} d N_{U}$ and $R_{T, \perp} d R_{T, \perp} \sim N_{T}^{2 \nu_{T}-1} d N_{T}$, with $\nu_{U} \simeq 0.6$ and $\nu_{T} \simeq 0.5$, where the longitudinal components of both $\mathrm{U}$ and $\mathrm{T}$ segments have been neglected in view of their much weaker dependence on the number of beads. The rate of work on the entire polymer, consisting of the $U$ and $T$ blobs with radii given by Eq.(6), takes then the form

$\frac{d W^{(1)}}{d t}=\left[\lambda_{T, \perp}^{2} N_{0}^{2 \nu_{T}} r^{2 \nu_{T}-1} \frac{d r}{d t}-\lambda_{U, \perp}^{2} N_{0}^{2 \nu_{U}}(1-r)^{2 \nu_{U}-1} \underset{d t}{d r}\right.$

with $r=N_{T}(t) / N_{0}$. Since $d W^{(1)} / d t$ is constant, integration of the above expression, with $r \in[0,1]$ and $t \in[0, \tau]$, leads to the scaling of the total translocation time: $\tau \sim N_{0}^{2 \nu_{U}}$. It is worth mentioning that, at variance with a previous argument [6], our macroscopic picture does not require that each blob be in a state of mechanical equilibrium. In fact, it is clear from Fig. 3 that at the end of translocation the blob is definitely not in equilibrium.

We next consider the viscous drag due to the net motion of the blob relative to the fluid, which can be computed by the global friction experienced by the whole set of $N_{0}$ monomers $F_{\text {drag }}=\sum_{i=1}^{N_{0}} \gamma\left(u_{i}-v_{i}\right)$, where again $v_{i}$ is the bead velocity, $u_{i}$ is the fluid velocity at the monomer location and $\gamma$ is the friction coefficient. This is best recast in the form of an effective friction coefficient $F_{\text {drag }}=-\gamma_{\text {eff }} \mathcal{V}$, where $\mathcal{V}$ is the center of mass velocity of the blob. As is well known [33], in the absence of hydrodynamic correlations all monomers behave independently, so that $\gamma_{\text {eff }}$ scales like $N_{0}$. On the other

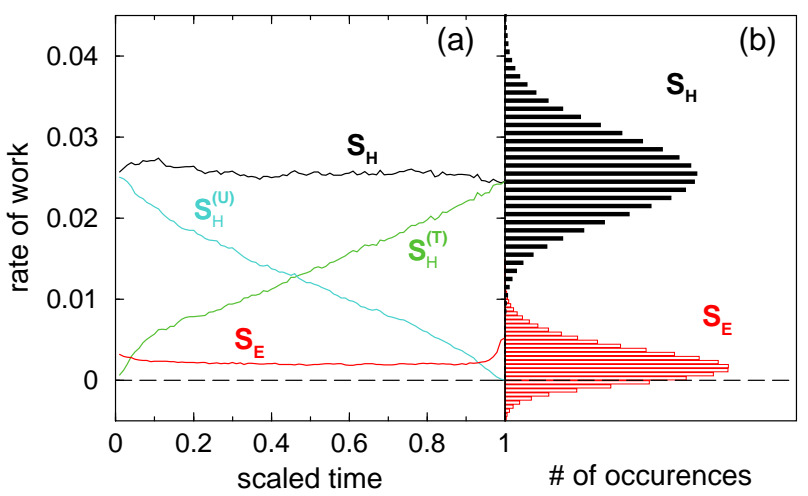

FIG. 5: (Color online) (a) Synergy factors with time for the hydrodynamic $\left(S_{H}^{(T)}, S_{H}^{(U)}\right.$, total $\left.S_{H}\right)$ and electric field $\left(S_{E}\right)$. (b) Probability distributions of $S_{H}, S_{E}$ during translocation events. Curves are averages over 100 events for $N_{0}=300$.

hand, when hydrodynamic correlations are included, the inner monomers are screened out from the outer ones, so that the effective friction is reduced and scales less than linearly with length. More precisely, $\gamma_{\text {eff }} \sim R \sim N_{0}^{\nu}$. Given that $\mathcal{V}=d R / d t$, the hydrodynamic drag scales like $N_{0}^{1+\nu}$ and $N_{0}^{2 \nu}$, without and with hydrodynamics, respectively. Remarkably, the exponent with hydrodynamics, $2 \nu$, is exactly the same as the one associated with the thermodynamic work $W^{(1)}$, so that $\alpha=2 \nu$ in either case. In the case without hydrodynamics, however, the thermodynamic work and the work due to viscous drag exhibit two distinct exponents, $2 \nu \simeq 1.2$ and $1+\nu \simeq 1.6$, which explains why any attempt to represent the scaling through a single exponent $\zeta$ is bound to work only on a narrow range of values of $N_{0}$. Generally, the scaling $d r \tau^{\tau}\left(N_{0}\right) \sim N_{0}^{\zeta}$ will be a weighted average of the two, i.e. $12 \nu<\zeta<1+\nu$

In support of the previous interpretation, we have measured the typical values of the rate of change of the blob radius $\dot{R}_{I}$, the mean center-mass velocity $\mathcal{V}$ and the average flow speed $\mathcal{U}$ and found that $\dot{R}_{I} \simeq \mathcal{V}_{I} \simeq 5 \mathcal{U}_{I}$. This corroborates the idea put forward in this paper according to which both mechanisms, the blob shrinking as well as the blob shifting processes, must be taken into account to provide a complete picture.

\section{DISCUSSION AND CONCLUSIONS}

Summarizing, we have investigated the process of polymer translocation through a narrow pore using a multiscale approach which explicitly accounts for the hydrodynamic interactions of the molecule with the surrounding solvent. The translocation time was found to obey a power-law dependence on the polymer length, with an exponent $\alpha=1.28 \pm 0.01$, in a satisfactory agreement with experimental measurements and other computer simulations. Moreover, our simulations reveal that the coupling 
of the molecular motion to hydrodynamic correlations results in a significant acceleration of the translocation process. The scaling behavior observed in the numerical simulations has been interpreted by means of a new phenomenological model, accounting for the anisotropy of both translocated and untranslocated segments. This ingredient appears to be crucial to the correct interpretation of the basic mechanisms behind the physics of the polymer translocation, which involves two separate processes, the shrinking and the shifting of the blob.

Deviations from the mean-field picture occur mainly near completion of the process, where the radius of the untranslocated segment undergoes an accelerated depletion: for $r(t)>1 / 2$, the majority of the beads have already translocated and entropic forces cooperate with the electric field to complete the translocation. Besides violating the static scaling at the end of the translocation process, entropic forces may lead to more dramatic effects, which escape any mean-field description based on the translocation coordinate $r(t)$ alone. Such beyondmean-field-theory effects produce rare retraction events: the polymer occasionaly anti-translocates after having partially passed through the pore. Our simulations reveal that retraction events are typically associated with the $T$ part entering a low-entropy (hairpin-like) configuration, which is then subject to a strong entropic pull-back. These non-perturbative events depend on the polymer length, the initial configuration and the values of other parameters (friction constant, temperature and strength of the pulling force). They occur at a rate up to $2 \%$ and do not significantly affect the statistics of the scaling exponent.

\section{Acknowledgments}

MF acknowledges support by Harvard's Nanoscale Science and Engineering Center, funded by NSF (Award No. PHY-0117795). SM and SS wish to thank the Physics Department at Harvard University for kind hospitality. We wish to thank H.C. Öttinger for valuable discussions.
[1] H. Lodish, D. Baltimore, A. Berk, S. Zipursky, P. Matsudaira, and J. Darnell, Molecular Cell Biology, W.H. Freeman \& Co, NY, (1996).

[2] J.J. Kasianowicz, E. Brandin, D. Branton, and D. Deamer, Proc. Nat. Acad. Sci. (USA) 93, 13770 (1996).

[3] A. Meller, L. Nivon, E. Brandin, J. Golovchenko, D. Branton, Proc. Nat. Acad. Sci. 97, 1079 (2000).

[4] C. Dekker, Nature Nanotech. 2, 209 (2007).

[5] J. Li, M. Gershow, D. Stein, E. Brandin, and J.A. Golovchenko, Nat. Mater. 2, 611 (2003).

[6] A.J. Storm, C. Storm, J. Chen, H. Zandbergen, J.-F. Joanny, and C. Dekker, Nanolett. 5, 1193 (2005).

[7] D.K. Lubensky and D.R. Nelson, Biophys. J. 77, 1824 (1999).

[8] W. Sung and P.J. Park, Phys. Rev. Lett. 77, 783 (1996).

[9] S. Ostojic and D. Panja, Phys. Rev. Lett. 97, 208001 (2006).

[10] J.L.A. Dubbeldam, A. Milchev, V.G. Rostiashvili, and T.A. Vilgis, Europhys. Lett. 79, 18002 (2007).

[11] S. Matysiak, A. Montesi, M. Pasquali, A.B. Kolomeisky, C. Clementi, Phys. Rev. Lett. 96, 118103 (2006).

[12] S. Kotsev and A. B. Kolomeisky, J. Chem. Phys. 127, 185103 (2007).

[13] I. Huopaniemi, K. Luo, and T. Ala-Nissila, J. Chem. Phys. 125, 124901 (2006).

[14] K. Luo, T. Ala-Nissila, S-C. Ying, A. Bhattacharya, Phys. Rev. Lett. 99, 148102 (2007).

[15] M.G. Fyta, S. Melchionna, E. Kaxiras, and S. Succi, Multiscale Model. \& Sim. 5, 1156 (2006).

[16] S. Melchionna, M.G. Fyta, E. Kaxiras, and S. Succi, Int. J. Mod. Phys. C 18, 685 (2007).

[17] C. Forrey and M. Muthukumar, J. Chem. Phys. 127, 015102 (2007).

[18] A. Izmitli, D.C. Schwartz, M.D. Graham, J. J. de Pablo,
J. Chem. Phys. 128, 085102 (2008).

[19] M.G. Gautier, G.W. Slater European Phys. J. E 25, 17 (2008); S. Guillouzic, G.W. Slater, Phys. Lett. A 359, 261 (2006).

[20] M. Bernaschi, S. Melchionna, S. Succi, M. Fyta, and E. Kaxiras, Nano Lett. (2008), DOI: 10.1021/nl073251f.

[21] R. Benzi, S. Succi, and M. Vergassola, Phys. Rep. 222, 145 (1992).

[22] S. Succi, O. Filippova, G. Smith, E. Kaxiras, Comput. Sci. and Eng. 3, 26 (2001).

[23] Y. Kantor and M. Kardar, Phys. Rev. E 69, 021806 (2004).

[24] J.P. Ryckaert et al, J. Comp. Phys. 23, 327 (1977).

[25] C.T.A. Wong and M. Muthukumar, J. Chem. Phys. 126, 164903 (2007).

[26] H.C. Öttinger, Stochastic Processes in Polymeric Fluids: Tools and Examples for Developing Simulation Algorithms, Springer, Berlin (1996).

[27] We thank H.C. Öttinger for bringing this point to our attention.

[28] J.D. Weeks, D. Chandler, and H.C. Andersen, J. Appl. Phys., 54, 5237 (1971).

[29] Y. Lu, B. Weers, and N.C. Stellwagen, Biopolymers. 61, 261 (2002).

[30] P. Ahlrichs, and B. Dünweg. J. Chem. Phys. 111, 8225 (1999).

[31] P.G. de Gennes, Scaling concepts in polymer physics, Cornell University Press, Ithaca NY, (1979).

[32] A.Milchev, V.Yamakov, K.Binder, Phys.Chem.Chem.Phys. 1, 2083 (1999).

[33] M. Doi, and S. F. Edwards, The Theory of Polymer Dynamics, Oxford University Press, Oxford, (1988). 